\title{
Quasi-partial $b$-metric spaces and some related fixed point theorems
}

\author{
Anuradha Gupta ${ }^{1}$ and Pragati Gautam²*
}

${ }^{\text {"Correspondence: }}$ pragati.knc@gmail.com

2Department of Mathematics, Kamala Nehru College, University of Delhi, August Kranti Marg, New Delhi, 110049, India

Full list of author information is available at the end of the article

\begin{abstract}
In this paper, the quasi-partial $b$-metric space is defined and general fixed point theorems on this space are discussed with examples.
\end{abstract}

MSC: 47H09; 47H10; 54H25

Keywords: quasi-metric space; partial-metric space; quasi-partial metric; T-orbitally lower semi-continuous; quasi-partial $b$-metric space; fixed point theorem

\section{Introduction}

A generalization of the metric space can be obtained as a partial-metric space by replacing the condition $d(x, x)=0$ with the condition $d(x, x) \leq d(x, y)$ for all $x, y$ in the definition of the metric. In the year 1993, Czerwik [1] introduced the concept of a $b$-metric space as another generalization of the concept of metric space. Several authors have focused on fixed point theorems for a metric space, a partial-metric space, quasi-partial metric space and a partial $b$-metric space. For further information on the subject see [2-16].

The concept of a quasi-partial-metric space was introduced by Karapınar et al. [17]. He studied some fixed point theorems on these spaces whereas Shatanawi and Pitea [18] studied some coupled fixed point theorems on quasi-partial-metric spaces.

The aim of this paper is to introduce the concept of quasi-partial $b$-metric spaces which is a generalization of the concept of quasi-partial-metric spaces. The fixed point results are proved in setting of such spaces and some examples are given to verify the effectiveness of the main results.

\section{Preliminaries}

We begin the section with some basic definitions and concepts.

Definition 2.1 ([17]) A quasi-partial metric on a non-empty set $X$ is a function $q: X \times$ $X \rightarrow \mathbb{R}^{+}$, satisfying

$\left(\mathrm{QPM}_{1}\right)$ If $q(x, x)=q(x, y)=q(y, y)$, then $x=y$.

$\left(\mathrm{QPM}_{2}\right) q(x, x) \leq q(x, y)$.

$\left(\mathrm{QPM}_{3}\right) q(x, x) \leq q(y, x)$.

$\left(\mathrm{QPM}_{4}\right) q(x, y)+q(z, z) \leq q(x, z)+q(z, y)$ for all $x, y, z \in X$.

A quasi-partial-metric space is a pair $(X, q)$ such that $X$ is a non-empty set and $q$ is a quasi-partial metric on $X$.

(C) 2015 Gupta and Gautam; licensee Springer. This is an Open Access article distributed under the terms of the Creative Commons Attribution License (http://creativecommons.org/licenses/by/4.0), which permits unrestricted use, distribution, and reproduction in any medium, provided the original work is properly credited. 
Let $q$ be a quasi-partial metric on the set $X$. Then

$$
d_{q}(x, y)=q(x, y)+q(y, x)-q(x, x)-q(y, y) \text { is a metric on } X .
$$

Lemma 2.1 ([17]) For a quasi-partial metric q on $X$,

$$
p_{q}(x, y)=\frac{1}{2}[q(x, y)+q(y, x)] \text { for all } x, y \in X \text { is a partial metric on } X \text {. }
$$

Lemmas $2.2([19-21])$

(A) A sequence $\left\{x_{n}\right\}$ is Cauchy in a partial-metric space $(X, p)$ if and only if $\left\{x_{n}\right\}$ is Cauchy in the (corresponding) metric space $\left(X, d_{p}\right)$.

(B) A partial-metric space $(X, p)$ is complete if and only if the (corresponding) metric space $\left(X, d_{p}\right)$ is complete. Moreover,

$$
\lim _{n \rightarrow \infty} d_{p}\left(x, x_{n}\right)=0 \quad \Leftrightarrow \quad p(x, x)=\lim _{n \rightarrow \infty} p\left(x, x_{n}\right)=\lim _{n, m \rightarrow \infty} p\left(x_{n}, x_{m}\right) .
$$

Lemma 2.3 ([17]) Let $(X, q)$ be a quasi-partial metric space, let $\left(X, p_{q}\right)$ be the corresponding partial-metric space, and let $\left(X, d_{p_{q}}\right)$ be the corresponding metric space. Then the following statements are equivalent:

(A) The sequence $\left\{x_{n}\right\}$ is Cauchy in $(X, q)$ and $(X, q)$ is complete.

(B) The sequence $\left\{x_{n}\right\}$ is Cauchy in $\left(X, p_{q}\right)$ and $\left(X, p_{q}\right)$ is complete.

(C) The sequence $\left\{x_{n}\right\}$ is Cauchy in $\left(X, d_{p_{q}}\right)$ and $\left(X, d_{p_{q}}\right)$ is complete. Also,

$$
\begin{aligned}
\lim _{n \rightarrow \infty} d_{q}\left(x, x_{n}\right)=0 \quad \Leftrightarrow \quad p_{q}(x, x) & =\lim _{n \rightarrow \infty} p_{q}\left(x, x_{n}\right)=\lim _{n, m \rightarrow \infty} p_{q}\left(x_{n}, x_{m}\right) \\
\Leftrightarrow \quad q(x, x) & =\lim _{n \rightarrow \infty} q\left(x, x_{n}\right)=\lim _{n, m \rightarrow \infty} q\left(x_{n}, x_{m}\right) \\
& =\lim _{n \rightarrow \infty} q\left(x_{n}, x\right)=\lim _{n, m \rightarrow \infty} q\left(x_{m}, x_{n}\right) .
\end{aligned}
$$

Definition 2.2 ([17]) If $T: X \rightarrow X$ is any map on $X, O(x)=\left\{x, T x, T^{2} x, \ldots\right\}$ is called the orbit of $x$. A mapping $G: X \rightarrow \mathbb{R}^{+}$is T-orbitally lower semi-continuous at $x$ if $\left\{x_{n}\right\}$ is a sequence in $O(x)$ and $\lim x_{n}=z$ implies $G(z) \leq \liminf G\left(x_{n}\right)$.

\section{Quasi-partial b-metric space}

We introduce the concept of quasi-partial $b$-metric space here.

Definition 3.1 A quasi-partial $b$-metric on a non-empty set $X$ is a mapping $q p_{b}: X \times X \rightarrow$ $\mathbb{R}^{+}$such that for some real number $s \geq 1$ and all $x, y, z \in X$ :

$\left(\mathrm{QPb}_{1}\right) q p_{b}(x, x)=q p_{b}(x, y)=q p_{b}(y, y) \Rightarrow x=y$,

$\left(\mathrm{QPb}_{2}\right) q p_{b}(x, x) \leq q p_{b}(x, y)$,

$\left(\mathrm{QPb}_{3}\right) q p_{b}(x, x) \leq q p_{b}(y, x)$

$\left(\mathrm{QPb}_{4}\right) q p_{b}(x, y) \leq s\left[q p_{b}(x, z)+q p_{b}(y, z)\right]-q p_{b}(z, z)$.

A quasi-partial b-metric space is a pair $\left(X, q p_{b}\right)$ such that $X$ is a non-empty set and $\left(X, q p_{b}\right)$ is a quasi partial $b$-metric on $X$. The number $s$ is called the coefficient of $\left(X, q p_{b}\right)$. 
For a quasi-partial $b$-metric space $\left(X, q p_{b}\right)$, the function $d_{q p_{b}}: X \times X \rightarrow \mathbb{R}^{+}$defined by

$$
d_{q p_{b}}(x, y)=q p_{b}(x, y)+q p_{b}(y, x)-q p_{b}(x, x)-q p_{b}(y, y) \text { is a } b \text {-metric on } X \text {. }
$$

Example 3.1 Let $X=[0,1]$.

Define $q p_{b}(x, y)=|x-y|+x$. Here

$$
q p_{b}(x, x)=q p_{b}(x, y)=q p_{b}(y, y) \quad \Rightarrow \quad x=y \quad \text { as } x=|x-y|+x=y \text { gives } x=y .
$$

Again, $q p_{b}(x, x) \leq q p_{b}(x, y)$ as $x \leq|x-y|+x$ and similarly, $q p_{b}(x, x) \leq q p_{b}(y, x)$ as $x \leq \mid y-$ $x \mid+y$ for $0<x<y$.

Also $q p_{b}(x, y)+q p_{b}(z, z) \leq s\left[q p_{b}(x, z)+q p_{b}(z, y)\right]$ as

$$
|x-y|+x+z \leq s[|x-z|+x+|z-y|+z] \text { for all } s \geq 1 \text {. }
$$

It can be observed that

$$
|x-y|+x+z=|x-z+z-y|+x+z \leq|x-z|+|z-y|+x+z .
$$

So $\left(X, q p_{b}\right)$ is a quasi-partial $b$-metric space with $s \geq 1$.

Example 3.2 Let $X=[1, \infty)$.

Define $q p_{b}: X \times X \rightarrow \mathbb{R}^{+}$as $q p_{b}(x, y)=\ln (x y)$. Then $\left(X, q p_{b}\right)$ is a quasi-partial $b$-metric space.

Let $q p_{b}(x, x)=q p_{b}(x, y)=q p_{b}(y, y) \Rightarrow \ln \left(x^{2}\right)=\ln (x y)=\ln \left(y^{2}\right) \Rightarrow x=y$.

Let $x, y \in X$. Without loss of generality $x \leq y \Rightarrow \ln x \leq \ln y \Rightarrow 2 \ln x \leq \ln x+\ln y \Rightarrow$ $\ln \left(x^{2}\right) \leq \ln x+\ln y$.

Thus, $q p_{b}(x, x) \leq q p_{b}(x, y)$.

Similarly $q p_{b}(x, x) \leq q p_{b}(y, x)$.

For $\left(\mathrm{QPb}_{4}\right)$ we have

$$
\begin{aligned}
q p_{b}(x, y) & =\ln x+\ln y \\
& \leq s \ln x+s \ln y \quad \text { since } s \geq 1 \text { and also } \ln x \geq 0 \text { and } \ln y \geq 0 \\
& \leq s \ln x+s \ln y+2 \ln z(s-1) \quad \text { since } \ln z \geq 0 \text { and } s-1 \geq 0 \\
& =s\left\{q p_{b}(x, z)+q p_{b}(z, y)\right\}-q p_{b}(z, z) .
\end{aligned}
$$

Example 3.3 Let $X=\left[0, \frac{\pi}{4}\right]$ and define $q p_{b}: X \times X \rightarrow \mathbb{R}^{+}$as

$$
q p_{b}(x, y)=\sin x+\sin y
$$

Then $\left(X, q p_{b}\right)$ is a quasi-partial $b$-metric space.

Lemma 3.4 Let $\left(X, q p_{b}\right)$ be a quasi-partial b-metric space. Then the following hold:

(A) If $q p_{b}(x, y)=0$ then $x=y$.

(B) If $x \neq y$, then $q p_{b}(x, y)>0$ and $q p_{b}(y, x)>0$. 
The proof is similar to the case of quasi-partial-metric space [17].

Lemma 3.5 Every quasi-partial space is a quasi-partial b-metric space. But the converse does not need to be true.

Definition 3.2 Let $\left(X, q p_{b}\right)$ be a quasi-partial $b$-metric. Then:

(i) A sequence $\left\{x_{n}\right\} \subset X$ converges to $x \in X$ if and only if

$$
q p_{b}(x, x)=\lim _{n \rightarrow \infty} q p_{b}\left(x, x_{n}\right)=\lim _{n \rightarrow \infty} q p_{b}\left(x_{n}, x\right)
$$

(ii) A sequence $\left\{x_{n}\right\} \subset X$ is called a Cauchy sequence if and only if

$$
\lim _{n, m \rightarrow \infty} q p_{b}\left(x_{n}, x_{m}\right) \text { and } \lim _{n, m \rightarrow \infty} q p_{b}\left(x_{m}, x_{n}\right) \text { exist (and are finite). }
$$

(iii) The quasi-partial $b$-metric space $\left(X, q p_{b}\right)$ is said to be complete if every Cauchy sequence $\left\{x_{n}\right\} \subset X$ converges with respect to $\tau_{q p_{b}}$ to a point $x \in X$ such that

$$
q p_{b}(x, x)=\lim _{n, m \rightarrow \infty} q p_{b}\left(x_{m}, x_{n}\right)=\lim _{n, m \rightarrow \infty} q p_{b}\left(x_{n}, x_{m}\right) .
$$

(iv) A mapping $f: X \rightarrow X$ is said to be continuous at $x_{0} \in X$ if, for every $\varepsilon>0$, there exists $\delta>0$ such that $f\left(B\left(x_{0}, \delta\right)\right) \subset B\left(f\left(x_{0}\right), \varepsilon\right)$.

Lemma 3.6 Let $\left(X, q p_{b}\right)$ be a quasi-partial b-metric space and $\left(X, d_{q p_{b}}\right)$ be the corresponding $b$-metric space. Then $\left(X, d_{q p_{b}}\right)$ is complete if $\left(X, q p_{b}\right)$ is complete.

Proof Since $\left(X, q p_{b}\right)$ is complete, every Cauchy sequence $\left\{x_{n}\right\}$ in $X$ converges with respect to $\tau_{q p_{b}}$ to a point $x \in X$ such that

$$
q p_{b}(x, x)=\lim _{n, m \rightarrow \infty} q p_{b}\left(x_{n}, x_{m}\right)=\lim _{n, m \rightarrow \infty} q p_{b}\left(x_{m}, x_{n}\right) .
$$

Consider a Cauchy sequence $\left\{x_{n}\right\}$ in $\left(X, d_{q p_{b}}\right)$. We will show that $\left\{x_{n}\right\}$ is Cauchy in $\left(X, q p_{b}\right)$. Since $\left\{x_{n}\right\}$ is Cauchy in $\left(X, d_{q p_{b}}\right), \lim _{n, m \rightarrow \infty} d_{q p_{b}}\left(x_{n}, x_{m}\right)$ exists and is finite.

$$
\text { Also, } d_{q p_{b}}\left(x_{n}, x_{m}\right)=q p_{b}\left(x_{n}, x_{m}\right)+q p_{b}\left(x_{m}, x_{n}\right)-q p_{b}\left(x_{n}, x_{n}\right)-q p_{b}\left(x_{m}, x_{m}\right) \text {. }
$$

Clearly, $\lim _{n, m \rightarrow \infty} q p_{b}\left(x_{n}, x_{m}\right)$ and $\lim _{n, m \rightarrow \infty} q p_{b}\left(x_{m}, x_{n}\right)$ exist and are finite.

Therefore, $\left\{x_{n}\right\}$ is a Cauchy sequence in $\left(X, q p_{b}\right)$. Now, since $\left(X, q p_{b}\right)$ is complete, the sequence $\left\{x_{n}\right\}$ converges with respect to $\tau_{q p_{b}}$ to a point $x \in X$ such that (1) holds.

For $\left\{x_{n}\right\}$ to be convergent in $\left(X, d_{q p_{b}}\right)$ we will show that $d_{q p_{b}}(x, x)=\lim _{n \rightarrow \infty} d_{q p_{b}}\left(x, x_{n}\right)$.

If follows from the definition of $d_{q p_{b}}$ that $d_{q p_{b}}(x, x)=0$. Also,

$$
\begin{aligned}
\lim _{n \rightarrow \infty} d_{q p_{b}}\left(x, x_{n}\right) & =\lim _{n \rightarrow \infty} q p_{b}\left(x, x_{n}\right)+\lim _{n \rightarrow \infty} q p_{b}\left(x_{n}, x\right)-\lim _{n \rightarrow \infty} q p_{b}\left(x_{n}, x_{n}\right)-\lim _{n \rightarrow \infty} q p_{b}(x, x) \\
& =0 \quad \text { by (1) and definition of convergence in }\left(X, q p_{b}\right) .
\end{aligned}
$$

Hence, $d_{q p_{b}}(x, x)=\lim _{n \rightarrow \infty} d_{q p_{b}}\left(x, x_{n}\right)$.

In [17] Karapinar et al. proved a fixed point theorem on quasi-partial-metric space. Motivated by this, we have generalized the results on a quasi-partial $b$-metric space. 


\section{The main results}

Theorem 4.1 Let $\left(X, q p_{b}\right)$ be a quasi-partial b-metric space, and let $T: X \rightarrow X$. Then the following hold:

(A) There exists $\phi: X \rightarrow \mathbb{R}^{+}$such that

$$
\begin{gathered}
q p_{b}(x, T x) \leq \phi(x)-\phi(T x) \quad \text { for all } x \in X \quad \text { if and only if } \\
\sum_{n=0}^{\infty} q p_{b}\left(T^{n} x, T^{n+1} x\right) \text { converges for all } x \in X .
\end{gathered}
$$

(B) There exists $\phi: X \rightarrow \mathbb{R}^{+}$such that

$$
\begin{gathered}
q p_{b}(x, T x) \leq \phi(x)-\phi(T x) \quad \text { for all } x \in O(x) \quad \text { if and only if } \\
\sum_{n=0}^{\infty} q p_{b}\left(T^{n} x, T^{n+1} x\right) \text { converges for all } x \in O(x) .
\end{gathered}
$$

Proof (A) Let $x \in X$, and let

$$
q p_{b}(x, T x) \leq \phi(x)-\phi(T x)
$$

Define the sequence $\left\{x_{n}\right\}_{n=1}^{\infty}$ in the following way:

$$
x_{0}=x \quad \text { and } \quad x_{n+1}=T x_{n}=T^{n+1} x_{0}, \quad \text { for all } n=0,1,2, \ldots
$$

Set $z_{n}(x)=\sum_{k=0}^{n} q p_{b}\left(x_{k}, x_{k+1}\right)=\sum_{k=0}^{n} q p_{b}\left(T^{k} x_{0}, T^{k+1} x_{0}\right)$. Then

$$
\begin{aligned}
z_{n}(x) & \leq \sum_{k=0}^{n}\left[\phi\left(T^{k} x_{0}\right)-\phi\left(T^{k+1} x_{0}\right)\right] \\
& =\left[\phi\left(x_{0}\right)-\phi\left(T x_{0}\right)\right]+\cdots+\left[\phi\left(T^{n} x_{0}\right)-\phi\left(T^{n+1} x_{0}\right)\right] \\
& =\left[\phi\left(x_{0}\right)-\phi\left(T^{n+1} x_{0}\right)\right] \leq \phi\left(x_{0}\right)=\phi(x)
\end{aligned}
$$

Thus, (2) implies that $\left\{z_{n}(x)\right\}$ is bounded. Also $\left\{z_{n}(x)\right\}$ is non-decreasing and hence convergent. Therefore, $\sum_{n=0}^{\infty} q p_{b}\left(T^{n} x, T^{n+1} x\right)$ converges.

Conversely, define

$$
\phi(x)=\sum_{n=0}^{\infty} q p_{b}\left(T^{n} x, T^{n+1} x\right) \quad \text { and } \quad z_{n}(x)=\sum_{k=0}^{n} q p_{b}\left(T^{k} x, T^{k+1} x\right) .
$$

Then

$$
\phi(T x)=\sum_{n=0}^{\infty} q p_{b}\left(T^{n+1} x, T^{n+2} x\right) \quad \text { and } \quad z_{n}(T x)=\sum_{k=0}^{n} q p_{b}\left(T^{k+1} x, T^{k+2} x\right) .
$$

Using these definitions, we get

$$
\begin{aligned}
z_{n}(x)-z_{n}(T x) & =\sum_{k=0}^{n} q p_{b}\left(T^{k} x, T^{k+1} x\right)-\sum_{k=0}^{n} q p_{b}\left(T^{k+1} x, T^{k+2} x\right) \\
& =q p_{b}(x, T x)-q p_{b}\left(T^{n+1} x, T^{n+2} x\right) .
\end{aligned}
$$


Since $\sum_{n=0}^{\infty} q p_{b}\left(T^{n} x, T^{n+1} x\right)$ converges for all $x \in X$,

$$
\lim _{n \rightarrow \infty} z_{n}(x)=\phi(x) \text { and } \quad \lim _{n \rightarrow \infty} q p_{b}\left(T^{n} x, T^{n+1} x\right)=0 .
$$

Letting $n \rightarrow \infty$ in (3) gives $q p_{b}(x, T x)=\phi(x)-\phi(T x)$.

(B) It can easily be proved using part (A).

Example 4.1 Let $X=[0,1]$. Define $q p_{b}(x, y)=|x-y|+|x|$.

Then $q p_{b}(x, y)$ satisfies all conditions of quasi-partial $b$-metric space. It is also quasipartial metric. But for $x \neq y, q p_{b}(x, y) \neq q p_{b}(y, x)$ and $q p_{b}(x, x) \neq 0$ for $x \neq 0$. So $q p_{b}$ is not a partial metric or a quasi-metric. Define $T: X \rightarrow X$ as $T x=\frac{x}{3}$ for all $x \in X$. Then the series $\sum_{n=0}^{\infty} q p_{b}\left(T^{n} x, T^{n+1} x\right)$ is convergent. Indeed,

$$
\begin{aligned}
\sum_{n=0}^{\infty} q p_{b}\left(T^{n} x, T^{n+1} x\right) & =\sum_{n=0}^{\infty} q p_{b}\left(\frac{x}{3^{n}}, \frac{x}{3^{n+1}}\right)=\sum_{n=0}^{\infty}\left|\frac{x}{3^{n}}-\frac{x}{3^{n+1}}\right|+\left|\frac{x}{3^{n}}\right| \\
& =\sum_{n=0}^{\infty}\left|\frac{2 x}{3^{n+1}}\right|+\left|\frac{x}{3^{n}}\right|=\sum_{n=0}^{\infty} \frac{5 x}{3^{n+1}}=\frac{5 x}{3} \cdot \frac{1}{1-\frac{1}{3}}=\frac{5 x}{2}
\end{aligned}
$$

Then the conditions of Theorem 4.1 are satisfied for $\phi(x)=\frac{5 x}{2}$. Indeed

$$
q p_{b}(x, T x)=q p_{b}\left(x, \frac{x}{3}\right)=\left|x-\frac{x}{3}\right|+|x|=\left|\frac{2 x}{3}\right|+|x|=\frac{5 x}{3}=\phi(x)-\phi(T x)
$$

The next result gives conditions for the existence of fixed points of operators on quasipartial $b$-metric space.

Theorem 4.2 Let $\left(X, q p_{b}\right)$ and $\left(Y, q p_{b}\right)$ be complete quasi-partial b-metric spaces. Let also $T: X \rightarrow X, R: X \rightarrow Y$, and $\phi: R(X) \rightarrow \mathbb{R}^{+}$. If there exist $x \in X$ and $c>0$ such that

$$
\max \left\{q p_{b}(y, T y), c q p_{b}(R y, R T y)\right\} \leq \phi(R y)-\phi(R T y)
$$

for all $y \in O(x)$, then the following hold:

(A) $\lim _{n \rightarrow \infty} T^{n} x=z$ exists.

(B) $T z=z$ if and only if $G(x)=q p_{b}(x, T x)$ is T-orbitally lower semi-continuous at $x$.

(C) $q p_{b}\left(x, T^{n} x\right) \leq s^{n-1} \phi(R x)$.

(D) For $m>n, q p_{b}\left(T^{n} x, T^{m} x\right) \leq s^{m-n}\left[\phi\left(R T^{n} x\right)\right]$.

Proof (A) Let $x \in X$. Define the sequence $\left\{x_{n}\right\}_{n=1}^{\infty}$ as follows:

$x_{0}=x \quad$ and $\quad x_{n+1}=T x_{n}=T^{n+1} x_{0}, \quad$ for all $n=0,1,2, \ldots$

We will show that $\left\{x_{n}\right\}_{n=1}^{\infty}$ is Cauchy.

Using $\left(\mathrm{QPb}_{4}\right)$, we get

$$
\begin{aligned}
q p_{b}\left(x_{n}, x_{n+2}\right) & \leq s\left\{q p_{b}\left(x_{n}, x_{n+1}\right)+q p_{b}\left(x_{n+1}, x_{n+2}\right)\right\}-q p_{b}\left(x_{n+1}, x_{n+1}\right) \\
& \leq s\left\{q p_{b}\left(x_{n}, x_{n+1}\right)+q p_{b}\left(x_{n+1}, x_{n+2}\right)\right\}
\end{aligned}
$$


and, similarly,

$$
\begin{aligned}
q p_{b}\left(x_{n}, x_{n+3}\right) & \leq s\left\{q p_{b}\left(x_{n}, x_{n+2}\right)+q p_{b}\left(x_{n+2}, x_{n+3}\right)\right\}-q p_{b}\left(x_{n+2}, x_{n+2}\right) \\
& \leq s^{2}\left\{q p_{b}\left(x_{n}, x_{n+1}\right)+q p_{b}\left(x_{n+1}, x_{n+2}\right)\right\}+s\left\{q p_{b}\left(x_{n+2}, x_{n+3}\right)\right\} .
\end{aligned}
$$

Now,

$$
\begin{aligned}
q p_{b}\left(x_{n}, x_{n+4}\right) \leq & s\left\{q p_{b}\left(x_{n}, x_{n+3}\right)+q p_{b}\left(x_{n+3}, x_{n+4}\right)\right\}-q p_{b}\left(x_{n+3}, x_{n+3}\right) \\
\leq & s^{3}\left\{q p_{b}\left(x_{n}, x_{n+1}\right)+q p_{b}\left(x_{n+1}, x_{n+2}\right)\right\}+s^{2}\left\{q p_{b}\left(x_{n+2}, x_{n+3}\right)\right\} \\
& +s\left\{q p_{b}\left(x_{n+3}, x_{n+4}\right)\right\} .
\end{aligned}
$$

On generalization, we get

$$
\begin{aligned}
q p_{b}\left(x_{n}, x_{m}\right) \leq & s^{m-n-1}\left\{q p_{b}\left(x_{n}, x_{n+1}\right)+q p_{b}\left(x_{n+1}, x_{n+2}\right)\right\} \\
& +s^{m-n-2}\left\{q p_{b}\left(x_{n+2}, x_{n+3}\right)\right\}+\cdots+s\left\{q p_{b}\left(x_{m-1}, x_{m}\right)\right\} \\
\leq & s^{m-n-1}\left\{q p_{b}\left(T^{n} x, T^{n+1} x\right)+q p_{b}\left(T^{n+1} x, T^{n+2} x\right)\right\} \\
& +s^{m-n-2}\left\{q p_{b}\left(T^{n+2} x, T^{n+3} x\right)\right\}+\cdots+s\left\{q p_{b}\left(T^{m-1} x, T^{m} x\right)\right\} \\
= & \sum_{k=n+1}^{m-1} s^{m-k}\left\{q p_{b}\left(T^{k} x, T^{k+1} x\right)\right\}+s^{m-n-1} q p_{b}\left(x_{n}, x_{n+1}\right) \\
= & \sum_{k=n}^{m-1} s^{m-k}\left\{q p_{b}\left(T^{k} x, T^{k+1} x\right)\right\}+s^{m-n-1} q p_{b}\left(x_{n}, x_{n+1}\right)-s^{m-n} q p_{b}\left(x_{n}, x_{n+1}\right) \\
= & \sum_{k=n}^{m-1} s^{m-k}\left\{q p_{b}\left(T^{k} x, T^{k+1} x\right)\right\}-s^{m-n} q p_{b}\left(x_{n}, x_{n+1}\right)\left[1-\frac{1}{s}\right] \\
\leq & \sum_{k=n}^{m-1} s^{m-k}\left\{q p_{b}\left(T^{k} x, T^{k+1} x\right)\right\} \quad \text { for } m>n .
\end{aligned}
$$

Set $z_{n}(x)=\sum_{k=0}^{n} s^{m-k}\left\{q p_{b}\left(T^{k} x, T^{k+1} x\right)\right\}$.

From (4) we have

$$
\begin{aligned}
s^{m-k}\left\{q p_{b}\left(T^{k} x, T^{k+1} x\right)\right\} & \leq s^{m-k} \max \left\{q p_{b}\left(T^{k} x, T^{k+1} x\right), c q p_{b}\left(R T^{k} x, R T^{k+1} x\right)\right\} \\
\leq & s^{m-k}\left\{\phi\left(R T^{k} x\right)-\phi\left(R T^{k+1} x\right)\right\} \quad \text { for all } k=0,1, \ldots \\
\Rightarrow \quad z_{n}(x) \leq & \sum_{k=0}^{n} s^{m-k}\left\{\phi\left(R T^{k} x\right)-\phi\left(R T^{k+1} x\right)\right\} \\
\leq & s^{m} \phi(R x)-s^{m} \phi(R T x)+s^{m} \phi(R T x)-s^{m-1} \phi\left(R T^{2} x\right)+\cdots \\
& +s^{m-n+1} \phi\left(R T^{n} x\right)-s^{m-n} \phi\left(R T^{n+1} x\right) \\
= & s^{m} \phi(R x)-s^{m-n} \phi\left(R T^{n+1} x\right) \\
\leq & s^{m} \phi(R x) .
\end{aligned}
$$


Thus, $\sum_{k=0}^{\infty} s^{m-k}\left\{q p_{b}\left(T^{k} x, T^{k+1} x\right)\right\}$ is convergent.

$$
\Rightarrow \quad \sum_{n=0}^{\infty} s^{m-n}\left\{q p_{b}\left(T^{n} x, T^{n+1} x\right)\right\} \text { is convergent. }
$$

Taking the limit as $n, m \rightarrow \infty$ in (7), we get

$$
\lim _{m, n \rightarrow \infty} q p_{b}\left(x_{n}, x_{m}\right)=\lim _{m, n \rightarrow \infty}\left(z_{m-1}(x)-z_{n-1}(x)\right)=0 .
$$

Using similar arguments,

$$
\lim _{m, n \rightarrow \infty} q p_{b}\left(x_{m}, x_{n}\right)=0
$$

Thus the sequence $\left\{x_{n}\right\}$ is Cauchy in $\left(X, q p_{b}\right)$. Since $\left(X, q p_{b}\right)$ is complete, $\left(X, d_{q p_{b}}\right)$ is also complete by Lemma 2.3, and hence $\lim _{n \rightarrow \infty} d_{q p_{b}}\left(T^{n} x, z\right)=0, \lim _{n \rightarrow \infty} T^{n} x=z$.

Further, $\lim _{n \rightarrow \infty} q p_{b}\left(T^{n} x, T^{n+1} x\right)=0$ and hence $\lim _{n \rightarrow \infty} q p_{b}\left(T^{n} x, T^{n+1} x\right)=q p_{b}(z, z)=0$.

(B) Assume that $T z=z$ and that $x_{n}$ is a sequence in $O(x)$ with $x_{n} \rightarrow z$.

By Lemma 3.6,

$$
\lim _{n \rightarrow \infty} d_{q p_{b}}\left(z, x_{n}\right)=0 \Leftrightarrow q p_{b}(z, z)=\lim _{n \rightarrow \infty} q p_{b}\left(z, x_{n}\right)=\lim _{n, m \rightarrow \infty} q p_{b}\left(x_{n}, x_{m}\right) .
$$

Then $G(z)=q p_{b}(z, T z)=q p_{b}(z, z) \leq \lim _{n \rightarrow \infty} \inf q p_{b}\left(x_{n}, T x_{n}\right)=\lim _{n \rightarrow \infty} \inf G\left(x_{n}\right)$.

Thus $G$ is $T$-orbitally lower semi-continuous at $x$.

Conversely, suppose that $x_{n}=T^{n} x \rightarrow z$ and that $G$ is $T$-orbitally lower semi-continuous at $x$. Then

$$
\begin{aligned}
0 & \leq q p_{b}(z, T z)=G(z) \leq \lim _{n \rightarrow \infty} \inf G\left(x_{n}\right)=\lim _{n \rightarrow \infty} \inf q p_{b}\left(T^{n} x, T^{n+1} x\right) \\
& =\lim _{n \rightarrow \infty} \inf q p_{b}\left(x_{n}, x_{n+1}\right)=q p_{b}(z, z)=0 .
\end{aligned}
$$

By Lemma 3.4, we have $T z=z$.

(C) We have, from $\left(\mathrm{QPb}_{4}\right)$ and (4),

$$
\begin{aligned}
q p_{b}\left(x, T^{2} x\right) & \leq s\left\{q p_{b}(x, T x)+q p_{b}\left(T x, T^{2} x\right)\right\}-q p_{b}(T x, T x) \\
& \leq s\left\{q p_{b}(x, T x)+q p_{b}\left(T x, T^{2} x\right)\right\} \\
q p_{b}\left(x, T^{3} x\right) & \leq s\left\{q p_{b}\left(x, T^{2} x\right)+q p_{b}\left(T^{2} x, T^{3} x\right)\right\}-q p_{b}\left(T^{2} x, T^{2} x\right) \\
& \leq s\left[s\left\{q p_{b}(x, T x)+q p_{b}\left(T x, T^{2} x\right)\right\}+q p_{b}\left(T^{2} x, T^{3} x\right)\right] \\
& \leq s^{2}\left\{q p_{b}(x, T x)+q p_{b}\left(T x, T^{2} x\right)\right\}+s\left\{q p_{b}\left(T^{2} x, T^{3} x\right)\right\}
\end{aligned}
$$

On generalization, we get

$$
\begin{aligned}
& q p_{b}\left(x, T^{n} x\right) \\
& \leq s^{n-1}\left\{q p_{b}(x, T x)+q p_{b}\left(T x, T^{2} x\right)\right\}+s^{n-2}\left\{q p_{b}\left(T^{2} x, T^{3} x\right)\right\}+\cdots \\
& \quad+s\left\{q p_{b}\left(T^{n-1} x, T^{n} x\right)\right\}
\end{aligned}
$$




$$
\begin{aligned}
\leq & s^{n-1}\left\{q p_{b}(x, T x)\right\}+s^{n-1}\left\{q p_{b}\left(T x, T^{2} x\right)\right\}+s^{n-2}\left\{q p_{b}\left(T^{2} x, T^{3} x\right)\right\}+\cdots \\
& +s\left\{q p_{b}\left(T^{n-1} x, T^{n} x\right)\right\} \\
\leq & s^{n-1}\{\phi(R x)-\phi(R T x)\}+s^{n-1}\left\{\phi(R T x)-\phi\left(R T^{2} x\right)\right\} \\
& +s^{n-2}\left\{\phi\left(R T^{2} x\right)-\phi\left(R T^{3} x\right)\right\}+\cdots+s\left\{\phi\left(R T^{n-1} x\right)-\phi\left(R T^{n} x\right)\right\} \\
\leq & s^{n-1} \phi(R x)-s^{n-1} \phi\left(R T^{2} x\right)+s^{n-2} \phi\left(R T^{2} x\right)-s^{n-2} \phi\left(R T^{3} x\right)+\cdots \\
& +s \phi\left(R T^{n-1} x\right)-s \phi\left(R T^{n} x\right) \\
\leq & s^{n-1} \phi(R x)-s \phi\left(R T^{2} x\right)-s \phi\left(R T^{n-1} x\right)-s \phi\left(R T^{n} x\right) \\
\leq & s^{n-1} \phi(R x) .
\end{aligned}
$$

(D) From (7) we get

$$
q p_{b}\left(x_{n}, x_{m}\right) \leq \sum_{k=n}^{m-1} s^{m-k}\left\{q p_{b}\left(T^{k} x, T^{k+1} x\right)\right\} \quad \text { for } m>n .
$$

Note that

$$
\begin{aligned}
\sum_{k=n}^{m-1} s^{m-k} q p_{b}\left(T^{k} x, T^{k+1} x\right) \\
\leq \sum_{k=n}^{m-1} s^{m-k}\left[\phi\left(R T^{k} x\right)-\phi\left(R T^{k+1} x\right)\right] \\
=s^{m-n} \phi\left(R T^{n} x\right)-s^{m-n} \phi\left(R T^{n+1} x\right)+s^{m-n-1} \phi\left(R T^{n+1} x\right) \\
\quad-s^{m-n-1} \phi\left(R T^{n+2} x\right)+\cdots+s \phi\left(R T^{m-1} x\right)-s \phi\left(R T^{m} x\right) \\
=s^{m-n} \phi\left(R T^{n} x\right)-s \phi\left(R T^{n+1} x\right)-s \phi\left(R T^{m-1} x\right)-s \phi\left(R T^{m} x\right) \\
\leq s^{m-n} \phi\left(R T^{n} x\right) .
\end{aligned}
$$

Here, $0 \leq q p_{b}\left(x_{n}, x_{m}\right)=q p_{b}\left(T^{n} x, T^{m} x\right) \leq s^{m-n} \phi\left(R T^{n} x\right)$ for $m>n$.

Example 4.2 Let $X=Y=[0,1]$. Define $q p_{b}(x, y)=|x-y|+x$. Then $q p_{b}$ is a quasi-partial $b$-metric with $s=1$. Also define $T: X \rightarrow X$ as $T(x)=\frac{x}{3} ; R: X \rightarrow Y$ as $R(x)=3 x$, and $\phi:$ $R(X) \rightarrow \mathbb{R}^{+}$as $\phi(x)=3 x$. Then for $c=1$ and $x \in[0,1]$ we have

$$
\begin{aligned}
\max \left\{q p_{b}(y, T y), c q p_{b}(R y, R T y)\right\} & =\max \left\{q p_{b}\left(y, \frac{y}{3}\right), q p_{b}(3 y, y)\right\} \\
& =\max \left\{\left|y-\frac{y}{3}\right|+y,|3 y-y|+3 y\right\} \\
& =\max \left\{\frac{5 y}{3}, 5 y\right\}=5 y<6 y=\phi(3 y)-\phi(y) \\
& =\phi(R y)-\phi(R T y) .
\end{aligned}
$$

We now prove that (A), (B), (C), and (D) of the above theorem hold:

(A) $\lim _{n \rightarrow \infty} T^{n} x=\lim _{n \rightarrow \infty} \frac{x}{3^{n}}=0=z$ (say). 
So $\lim _{n \rightarrow \infty} T^{n} x=z$ exists.

(B) By (A) part above, $z=0$.

Therefore $T(z)=T(0)=0=z$ holds trivially.

Hence whenever $G(x)=q p_{b}(x, T x)$ is $T$-orbitally lower semi-continuous at $x$ then $T z=z$.

Conversely, let $T z=z$ and we show that $G$ is $T$-orbitally lower semi-continuous at $x$, i.e.,

$$
G(z) \leq \liminf G\left(x_{n}\right) \quad \forall\left\{x_{n}\right\} \subseteq O(x), x_{n} \rightarrow z .
$$

Let $\left\{x_{n}\right\} \subseteq O(x)$ be a sequence converging to $z$. Then

$$
\begin{aligned}
G(z) & =q p_{b}(z, T z)=q p_{b}(z, z)=z \\
& =\frac{5 z}{3}(\operatorname{as} z=0)=\liminf \frac{5 x_{n}}{3} \\
& =\liminf \frac{2 x_{n}}{3}+x_{n}=\liminf \left|x_{n}-\frac{x_{n}}{3}\right|+x_{n} \\
& =\liminf q p_{b}\left(x_{n}, \frac{x_{n}}{3}\right)=\liminf q p_{b}\left(x_{n}, T x_{n}\right)=\liminf G\left(x_{n}\right) .
\end{aligned}
$$

Hence $G(z)=\liminf G\left(x_{n}\right)$.

$$
\begin{aligned}
\text { (C) } q p_{b}\left(x, T^{n} x\right) & =q p_{b}\left(x, \frac{x}{3^{n}}\right)=\left|x-\frac{x}{3^{n}}\right|+x=x\left(2-\frac{1}{3^{n}}\right)<x(9) \quad \forall n \in N \\
& =\phi(3 x)=s^{n-1} \phi(R x) \quad \text { where } s=1 .
\end{aligned}
$$

(D) Let $m>n$ then

$$
\begin{aligned}
q p_{b}\left(T^{n} x, T^{m} x\right) & =q p_{b}\left(\frac{x}{3^{n}}, \frac{x}{3^{m}}\right)=\left|\frac{x}{3^{n}}-\frac{x}{3^{m}}\right|+\frac{x}{3^{n}} \\
& =\frac{x}{3^{n}}\left[2-\frac{1}{3^{m-n}}\right]<\frac{x}{3^{n}}(9) \quad \forall n \in N \\
& =\phi\left(\frac{x}{3^{n-1}}\right)=\phi\left(3 T^{n} x\right)=s^{m-n}\left[\phi\left(R T^{n} x\right)\right] \quad \text { where } s=1 .
\end{aligned}
$$

Corollary 4.3 Let $\left(X, q p_{b}\right)$ be a complete quasi-partial b-metric space. Let $T: X \rightarrow X$ and $\phi: X \rightarrow \mathbb{R}^{+}$. Suppose that there exists $x \in X$ such that

$$
q p_{b}(y, T y) \leq \phi(y)-\phi(T y) \quad \text { for all } y \in O(x)
$$

\section{Then the following hold:}

(A) $\lim _{n \rightarrow \infty} T^{n} x=z$ exists.

(B) $T z=z$ if and only if $G(x)=q p_{b}(x, T x)$ is T-orbitally lower semi-continuous at $x$.

(C) $q p_{b}\left(x, T^{n} x\right) \leq s^{n-1} \phi(x)$.

(D) For $m>n, q p_{b}\left(T^{n} x, T^{m} x\right) \leq s^{m-n} \phi\left(T^{n} x\right)$.

Proof Take $Y=X, R=I$, and $c=1$ in Theorem 4.2. 
Corollary 4.4 Let $\left(X, q p_{b}\right)$ be a complete quasi-partial b-metric space, and let $0<k<1$. Suppose that $T: X \rightarrow X$ and that there exists $x \in X$ such that

$$
q p_{b}\left(T y, T^{2} y\right) \leq k q p_{b}(y, T y) \quad \text { for all } y \in O(x)
$$

Then the following hold:

(A) $\lim _{n \rightarrow \infty} T^{n} x=z$ exists.

(B) $T z=z$ if and only if $G(x)=q p_{b}(x, T x)$ is T-orbitally lower semi-continuous at $x$.

(C) $q p_{b}\left(x, T^{n} x\right) \leq \frac{s^{n-1}}{1-k} q p_{b}(x, T x)$.

Proof Set $\phi(y)=\frac{1}{1-k} q p_{b}(y, T y)$ for $y \in O(x)$.

Let $y=T^{n} x$ in (16). Then

$$
q p_{b}\left(T^{n+1} x, T^{n+2} x\right) \leq k q p_{b}\left(T^{n} x, T^{n+1} x\right)
$$

and

$$
q p_{b}\left(T^{n} x, T^{n+1} x\right)-k q p_{b}\left(T^{n} x, T^{n+1} x\right) \leq q p_{b}\left(T^{n} x, T^{n+1} x\right)-q p_{b}\left(T^{n+1} x, T^{n+2} x\right) .
$$

Thus, $q p_{b}\left(T^{n} x, T^{n+1} x\right) \leq \frac{1}{1-k}\left[q p_{b}\left(T^{n} x, T^{n+1} x\right)-q p_{b}\left(T^{n+1} x, T^{n+2} x\right)\right]$ or $q p_{b}(y, T y) \leq[\phi(y)-$ $\phi(T y)]$.

(A)-(C) follow immediately from Corollary 4.3.

Corollary 4.5 Let $\left(X, q p_{b}\right)$ be a complete quasi-partial b-metric space where $q p_{b}$ is continuous. Let $T: X \rightarrow X$ and $\phi: X \rightarrow \mathbb{R}^{+}$is continuous. Suppose that there exists $x \in X$ such that

$$
q p_{b}(y, T y) \leq \phi(y)-\phi(T y) \quad \text { for all } y \in O(x) .
$$

Then the following hold:

(A) $\lim _{n \rightarrow \infty} T^{n} x=z$ exists.

(B) $q_{p}(z, z) \leq s \phi(z)$.

Proof In Theorem 4.2(D) taking $m=n+1, R=I, c=1$, and $Y=X$,

$$
q p_{b}\left(T^{n} x, T^{n+1} x\right) \leq s\left[\phi\left(R T^{n} x\right)\right] .
$$

Now taking $\lim n \rightarrow \infty$

$$
\begin{aligned}
& \lim _{n \rightarrow \infty} q p_{b}\left(T^{n} x, T^{n+1} x\right) \leq \lim _{n \rightarrow \infty} s\left[\phi\left(T^{n} x\right)\right], \\
& q p_{b}(z, z) \leq s \phi(z) .
\end{aligned}
$$


Authors' contributions

Both authors contributed equally and significantly in writing this article. Both authors read and approved the final manuscript.

\section{Author details}

1 Department of Mathematics, Delhi College of Arts and Commerce, University of Delhi, New Delhi, 110023, India.

2 Department of Mathematics, Kamala Nehru College, University of Delhi, August Kranti Marg, New Delhi, 110049 , India.

Received: 28 August 2014 Accepted: 2 January 2015 Published online: 03 February 2015

\section{References}

1. Czerwik, S: Contraction mappings in b-metric spaces. Acta Math. Inform. Univ. Ostrav. 1, 5-11 (1993)

2. Mukheimer, A: $\alpha-\psi-\phi$-Contractive mappings in ordered partial $b$-metric spaces. J. Nonlinear Sci. Appl. 7, 168-179 (2014)

3. Shatanawi, W: On $\omega$-compatible mappings and common coupled coincidence point in cone metric spaces. Appl. Math. Lett. 25, 925-931 (2012)

4. Bhaskar, TG, Lakshmikantham, V: Fixed point theorems in partially ordered metric spaces and applications. Nonlinear Anal. 65, 1379-1393 (2006)

5. Lakshmikantham, V, Ćirić, L: Coupled fixed point theorems for nonlinear contractions in partially ordered metric spaces. Nonlinear Anal. 70, 4341-4349 (2009)

6. Hicks, TL: Fixed point theorems for quasi-metric spaces. Math. Jpn. 33(2), 231-236 (1988)

7. Karapınar, E: Generalizations of Caristi Kirk's theorem on partial metric spaces. Fixed Point Theory Appl. 2011, Article ID 4 (2011)

8. Ali, MU: Mizoguchi-Takahashi's type common fixed point theorem. J. Egypt. Math. Soc. 22, 272-274 (2014)

9. Bakhtin, IA: The contraction principle in quasimetric spaces. In: Functional Analysis, vol. 30, pp. 26-37 (1989)

10. Bota, M-F, Karapınar, E, Mleşniţe, O: Ulam-Hyers stability results for fixed point problems via $\alpha$ - $\psi$-contractive mapping in (b)-metric space. Abstr. Appl. Anal. 2013, Article ID 825293 (2013)

11. Bota, M-F, Karapınar, E: A note on 'Some results on multi-valued weakly Jungck mappings in $b$-metric space'. Cent. Eur. J. Math. 11(9), 1711-1712 (2013)

12. Aydi, H, Bota, M-F, Karapınar, E, Moradi, S: A common fixed point for weak $\phi$-contractions ON $b$-metric spaces. Fixed Point Theory 13(2), 337-346 (2012)

13. Aydi, H, Bota, M-F, Karapınar, E, Mitrović, S: A fixed point theorem for set-valued quasi-contractions in $b$-metric spaces. Fixed Point Theory Appl. 2012, Article ID 88 (2012)

14. Latif, A, Al-Mezel, SA: Fixed point results in quasi metrics spaces. Fixed Point Theory Appl. 2011, Article ID 178306 (2011)

15. Shukla, S: Partial b-metric spaces and fixed point theorems. Mediterr. J. Math. 11, 703-711 (2014)

16. Caristi, J: Fixed point theorems for mapping satisfying inwardness conditions. Trans. Am. Math. Soc. 215, 241-251 (1976)

17. Karapınar, E, Erhan, ÍM, Özturk, A: Fixed point theorems on quasi-partial metric spaces. Math. Comput. Model. 57, 2442-2448 (2013)

18. Shatanawi, W, Pitea, A: Some coupled fixed point theorems in quasi-partial metric spaces. Fixed Point Theory Appl. 2013. Article ID 153 (2013). doi:10.1186/1637-1812-2013-153

19. Matthews, SG: Partial metric topology, general topology and its applications. Ann. N.Y. Acad. Sci. 728, 183-197 (1994)

20. Altun, I, Erduran, A: Fixed point theorems, for monotone mappings on partial metric spaces. Fixed Point Theory Appl. 2011, Article ID 508730 (2011)

21. Matthews, SG: Partial Metric Topology. Research Report 212, Department of Computer Science, University of Warwick (1992)

\section{Submit your manuscript to a SpringerOpen ${ }^{\circ}$ journal and benefit from:}

- Convenient online submission

- Rigorous peer review

- Immediate publication on acceptance

- Open access: articles freely available online

- High visibility within the field

- Retaining the copyright to your article 